\title{
Zinc Cobalt Layered Double Hydroxide Electrode for High-Performance Supercapacitor
}

\author{
Received August 1, 2019; accepted August 22, 2019
}

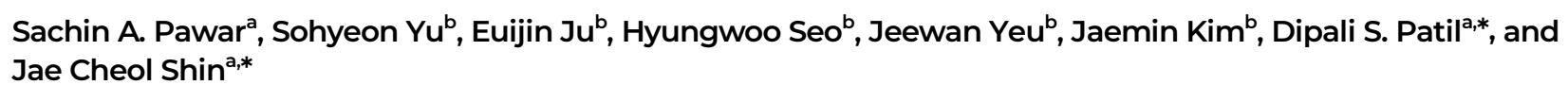

aDepartment of Physics, Yeungnam University, Gyeongsan 38541, Republic of Korea

bDaegu Science High School, Daegu 42110, Republic of Korea

*Corresponding author E-mail: dipalipatilphy18@ynu.ac.kr, jcshin@yu.ac.kr

\begin{abstract}
We aim to fabricate a ZnCo layered double hydroxide (LDH) nanostructure through facile one-pot hydrothermal synthesis. ZnCo LDH with interconnected hexagonal sheets having ultrathin thickness with voids are synthesized for the effective penetration of electrolytes to achieve superior electrochemical properties. The as-synthesized ZnCo LDH nanostructure is then subjected to structural, morphological, phase, and electrochemical studies. As an electrode for a supercapacitor, the ZnCo LDH has an areal capacitance of $1964 \mathrm{mFcm}^{-2}$ at a $5 \mathrm{mVs}^{-1}$ scan rate, an energy density of $0.22 \mathrm{mWhcm}^{-2}$, and an excellent cycling stability (112\%) after 2000 charge-discharge cycles.
\end{abstract}

Keywords: ZnCo, Layered double hydroxide, Supercapacitor

\section{Introduction}

Modern society is technologically advanced and most daily electronic appliances are energy dependent. It is essential to have high-efficiency energy storage systems to fulfill the increasing demand for renewable energy. Supercapacitors, which are also called electrochemical supercapacitors, are gaining considerable attention as a viable energy storage system owing to their long cycle life, high-power density, and rapid recharge ability [1]. There are two types of supercapacitors based on their charge storage mechanisms: electric double layer capacitors (EDLCs) and pseudocapacitors. In EDLCs, the charge is stored at the electrolyte/electrode interface [2]. Various carbon-based materials possessing high surface area are widely used for EDLCs applications, such as graphene, activated carbon, and carbon nanotubes (CNTs) based electrodes [3]. In a pseudocapacitor, the charge is stored due to Faradaic redox reactions at the electrode surface leading to pseudocapacitance. The pseudocapacitance behavior is found in different transition metal oxides (TMOs) and conducting polymers [4].

In pseudocapacitors, various TMOs are widely used because of their high specific capacitance than the carbon-based materials. Unfortunately, the low electronic conductivity of the binary TMOs leads to poor electrochemical activity, which limits their practical application [5]. On the other hand, carbon-based materials have a serious drawback such as poor energy density, which cannot be utilized to meet the requirements of modern electronics, and have limitation related to commercialization. Therefore, it is necessary to develop and synthesize novel low-cost materials possessing high energy density and long cycling stability for efficient energy storage systems.

Recently, layered double hydroxides (LDHs) with nanostructures have received considerable attention in studies on electrochemical supercapacitors [6]. It has been proved in the literature that LDH electrodes possess richer Faradaic reactions and enhanced capacitances owing to an improvement in pseudocapacitive properties. LDHs are also known as hydrotalcite-like compounds, which are inorganic layered materials whose structure is based on brucite-like layers. The general formula of an LDH is $\left.\left[M_{1-x}^{I I} M_{x}^{I I I}(O H)_{2}\right]^{Z+}\left(A^{n-}\right)_{\frac{Z}{n}} \cdot \gamma H_{2} O\right]$, where $M^{I I}$ and $M^{I I I}$ are divalent and trivalent metals, respectively, and $A^{n-}$ is the interlayer anion [7]. The beneficial properties of LDHs include their tunable composition, layered structure, highly dispersive cations in host layers, and high specific capacitance. Owing to these properties, LDHs have been widely exploited in supercapacitor studies [8-10].

In this work, we fabricate a $\mathrm{ZnCo}$ based $\mathrm{LDH}$ for realizing a superior supercapacitor by using facile one-pot hydrothermal synthesis. It is known that $\mathrm{Zn}$ resources are abundant in nature, possess outstanding Faradaic pseudocapacitive performance, and are rich in redox reactions of $\mathrm{Zn}$-Co compounds. Hence, bimetallic $\mathrm{Zn}$-Co hydroxides are considered highly suitable for future use in supercapacitors; nanostructures of $\mathrm{Zn}$-Co hydroxides synthesized with an easy route are highly promising. The optimized electrode of $\mathrm{ZnCo} \mathrm{LDH}$ is subjected to structural, morphological, phase, and electrochemical studies. It is found that the nanostructures of $\mathrm{ZnCo} \mathrm{LDH}$ show promising electrochemical properties, exhibiting an areal capacitance of $1964 \mathrm{mFcm}^{-2}$ at a $5 \mathrm{mVs}^{-1}$ scan rate, with an excellent capacitance retention of $112 \%$ after 2000 cycles, which is superior than the properties of other metallic LDH nanostructures. 


\section{Experimental details}

$\mathrm{Zn}$-Co hydroxide was grown on $\mathrm{Ni}$ foam by a hydrothermal synthesis procedure. Zinc acetate dihydrate $\left(\mathrm{Zn}\left(\mathrm{CH}_{3} \mathrm{COO}\right)_{2} \cdot 2 \mathrm{H}_{2} \mathrm{O}\right)$, cobalt nitrate hexahydrate $\left(\mathrm{Co}\left(\mathrm{NO}_{3}\right)_{2}\right) \cdot 6 \mathrm{H}_{2} \mathrm{O}$, and urea $\left(\mathrm{CO}\left(\mathrm{NH}_{2}\right)_{2}\right)$ were used as source materials without further purification. In a typical procedure, $0.01 \mathrm{M}$ of $\left(\mathrm{Zn}\left(\mathrm{CH}_{3} \mathrm{COO}\right)_{2} \cdot 2 \mathrm{H}_{2} \mathrm{O}\right), 0.02 \mathrm{M}$ of $\left(\mathrm{Co}\left(\mathrm{NO}_{3}\right)_{2}\right) \cdot$ $6 \mathrm{H}_{2} \mathrm{O}$, and $0.05 \mathrm{M}$ of $\left(\mathrm{CO}\left(\mathrm{NH}_{2}\right)_{2}\right)$ were dissolved in $60 \mathrm{~mL}$ of distilled water and kept under constant stirring conditions. The resulting homogeneous solution $(60 \mathrm{~mL})$ was then transferred to an $80-\mathrm{mL}$ Teflon lined stainless steel autoclave. A piece of cleaned Ni foam was immersed in the solution by inclining it against the wall of the Teflon liner. Then, the autoclave was sealed and placed in an oven at $140{ }^{\circ} \mathrm{C}$ for $8 \mathrm{~h}$. Finally, the Ni foam coated with the resultant product was removed from the autoclave after cooling to room temperature and rinsed thoroughly using distilled water and kept at room temperature for air drying. Thus, the obtained electrode is referred as $\mathrm{ZnCo} \mathrm{LDH}$.

\section{Results and discussion}

The ZnCo LDH electrode fabricated by the 8-h hydrothermal reaction was characterized to assess the properties of the material. $\mathrm{X}$-ray diffraction (XRD) was used to study the phase and crystal structure of the $\mathrm{ZnCo} \mathrm{LDH}$ sample. Figure 1 shows the XRD pattern of the $\mathrm{ZnCo} \mathrm{LDH}$ electrode coated on nickel (Ni) foam. The

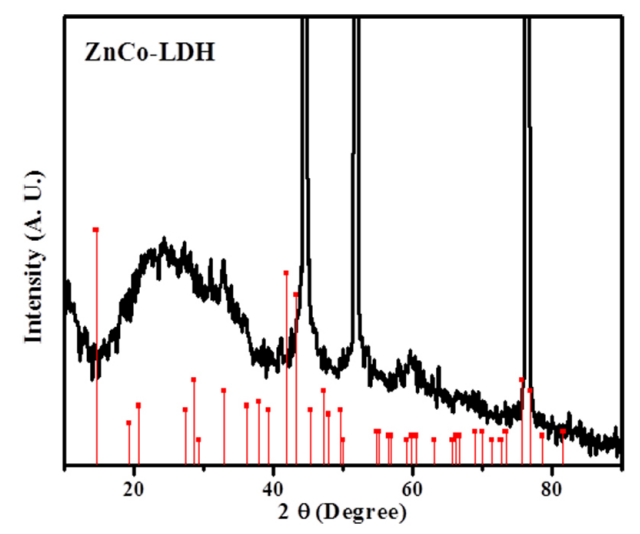

Figure 1. (Color online) XRD pattern of $\mathrm{ZnCo}-\mathrm{LDH}$. diffraction peaks located at $2 \theta$ values of $14.73^{\circ}, 19.39^{\circ}, 28.65^{\circ}, 29.45^{\circ}$, $32.99^{\circ}, 36.31^{\circ}, 37.75^{\circ}, 42.09^{\circ}, 47.51^{\circ}$, and $49.67^{\circ}$ were assigned to the (002), (011), (013), (111), (-113), (020), (113), (200), (024), and (-124) planes of the $\mathrm{ZnCo} \mathrm{LDH}$ nanostructure, respectively. These diffracted peaks are in accordance with the standard data file JCPDS no. 21-1477 [11]. Along with these diffraction peaks, the peaks located at $44.55^{\circ}$, $51.91^{\circ}$, and $76.47^{\circ}$ were caused by the substrate $\mathrm{Ni}$ foam.

To obtain information on the chemical state and valency of a material, X-ray photoelectron spectroscopy (XPS) measurement was performed. Figures $2(\mathrm{a})-(\mathrm{c})$ show the high resolution XPS of a sample $\mathrm{ZnCo} \mathrm{LDH}$ nanostructure consisting of $\mathrm{Zn} 2 \mathrm{p}$, Co $2 \mathrm{p}$, and $\mathrm{O} 1 \mathrm{~s}$ spectra. In the $\mathrm{Zn} 2 \mathrm{p}$ spectrum, the binding energy peaks located at 1024.08 and $1047.28 \mathrm{eV}$ correspond to $\mathrm{Zn} 2 \mathrm{p}_{3 / 2}$ and $\mathrm{Zn} 2 \mathrm{p}_{1 / 2}$, respectively. The characteristic binding energy peak at $\sim 1024 \mathrm{eV}$ is caused by $\mathrm{Zn}^{2+}$ in the metal hydroxide, and it confirms that the valency of $\mathrm{Zn}$ is +2 [12]. Similarly, the Co $2 \mathrm{p}$ XPS spectra consists of two main peaks Co $2 \mathrm{p}_{3 / 2}$ and Co $2 \mathrm{p}_{1 / 2}$ located at 783.28 and $799.38 \mathrm{eV}$, which can be attributed to $\mathrm{Co}^{3+}$ and $\mathrm{Co}^{2+}$, respectively. The O 1s XPS spectrum consists of three peaks located at 529.58, 531.28, and 534.38 $\mathrm{eV}$, which are caused by Co-O, M-OH bonding (where $\mathrm{M}=\mathrm{Zn}, \mathrm{Co}$ ), and surface contamination, respectively [13]. From the XPS analysis, it is observed that the $\mathrm{ZnCo} \mathrm{LDH}$ material is composed of $\mathrm{Zn}$, Co, and $\mathrm{OH}$ without any impurities, thus affirming the purity of our sample.

The surface morphological features of the sample $\mathrm{ZnCo} \mathrm{LDH}$ were determined through field emission scanning electron microscopy (FESEM). Figures 3(a)-(f) show the FESEM images of ZnCo $\mathrm{LDH}$ at different magnifications. It is clearly observed that the entire $\mathrm{Ni}$ foam is coated uniformly with the $\mathrm{ZnCo} \mathrm{LDH} \mathrm{nanostructures,} \mathrm{as}$ seen in Fig. 3(a). After viewing the morphology at further higher magnifications [Figs. 3(b) and 3(c)], it is interesting to note that the $\mathrm{ZnCo} \mathrm{LDH}$ nanostructure is composed of hexagonal sheets interconnected with each other. This interconnected network of hexagonal sheets has resulted in various valley points resembling voids that are highly beneficial for the effective penetration of the liquid electrolyte leading to enhanced electrochemical activity. FESEM images at magnifications of $\times 25, \times 50$, and $\times 100 \mathrm{~K}$ [Figs. $3(\mathrm{~d})-(\mathrm{f})]$ show that the hexagonal sheets of $\mathrm{ZnCo} \mathrm{LDH}$ are stacked upon each other and the thickness of each hexagonal sheet is $\sim 60 \mathrm{~nm}$.

To investigate the electrochemical performance of the as prepared $\mathrm{ZnCo}-\mathrm{LDH}$, electrochemical measurements are performed through conventional three electrodes configurations in $2 \mathrm{M} \mathrm{KOH}$. Figure 4(a) shows a CV curve of $\mathrm{ZnCo-LDH}$ at a scan rate of $5 \mathrm{mVs}^{-1}$ within the
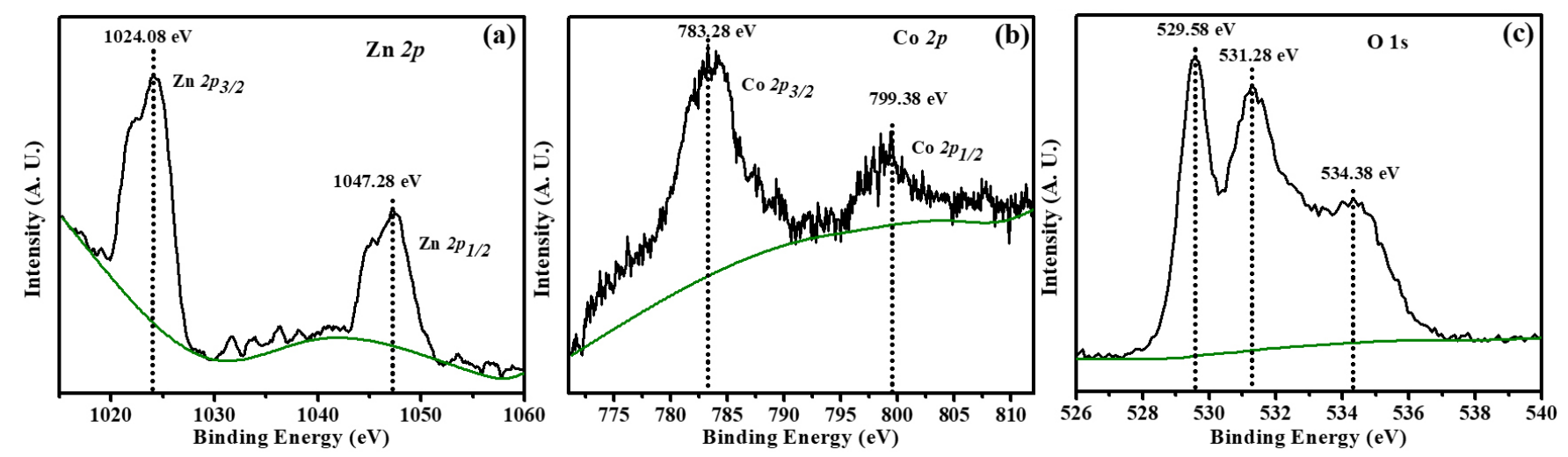

Figure 2. (Color online) High-resolution XPS of ZnCo-LDH: (a) Zn 2p, (b) Co 2p, (c) 01 s. 


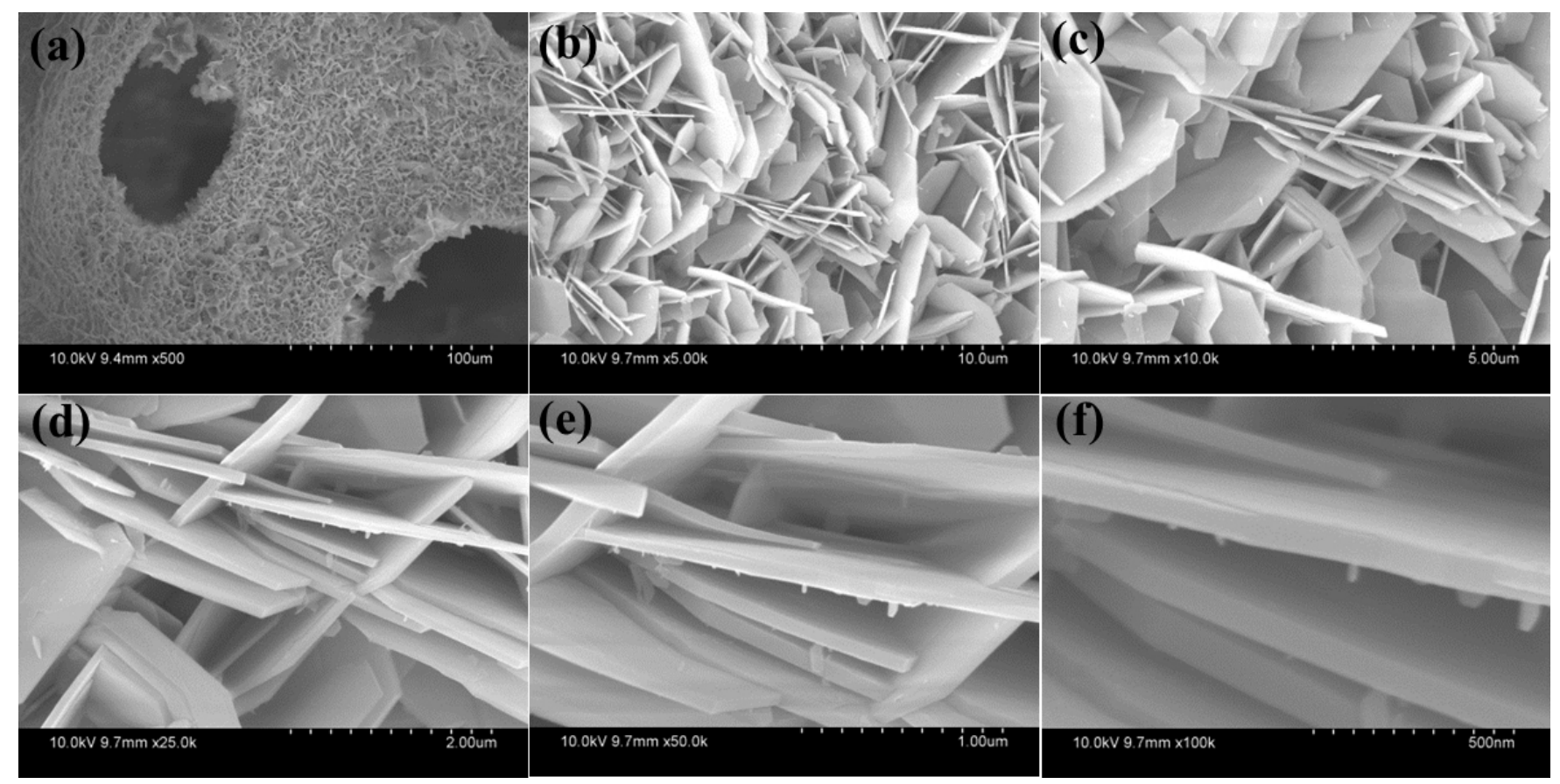

Figure 3. SEM images of ZnCo-LDH at different magnifications.
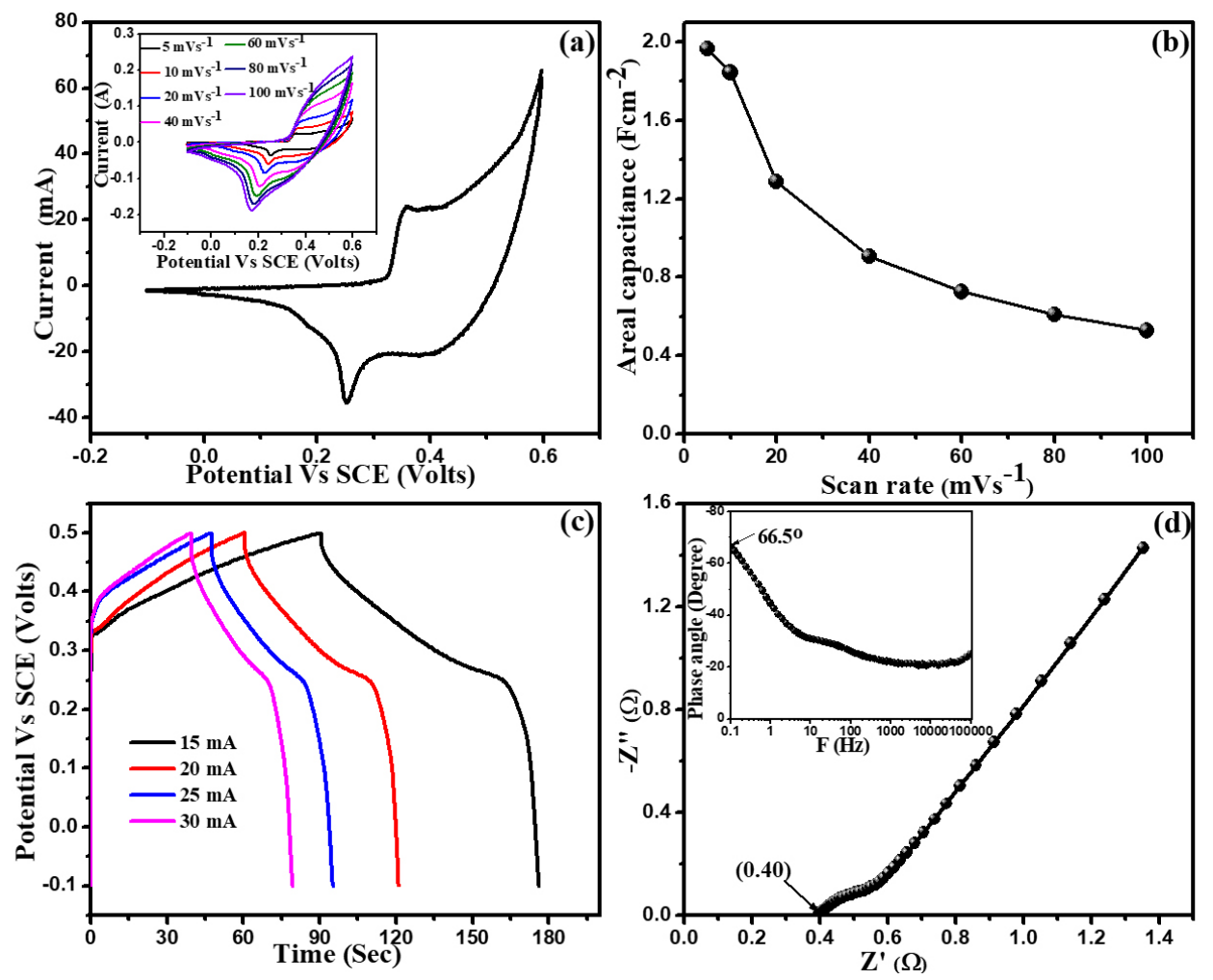

Figure 4. (Color online) (a) $\mathrm{CV}$ curves at a scan rate of $5 \mathrm{mVs}^{-1}$, Inset: at different scan rate, (b) charge-discharge at different applied current, (c) areal capacitance versus scan rate, (d) Nyquist plot, Inset: magnified Nyquist plots of ZnCo-LDH.

potential range between -0.1 and $0.6 \mathrm{~V}$ vs. Saturated Calomel Electrode (SCE). From the CV curve, two strong redox peaks can be clearly seen, which are attributed to faradaic reactions occurring between the active material and the electrolyte. This signifies the pseudo-capacitive charge storage mechanism of the synthesized electrode. The inset of Fig. 4(a) exhibits the CV curves of $\mathrm{ZnCo-LDH}$ recorded at different 
scan rates. As the scan rate increases, the area under the CV curves increases, and this is correlated with the linear variation of the peak current with the square root of the scan rate. The areal capacitance of the electrode was calculated from the CV curve integrals by

$$
C_{A}=\frac{\int i d V}{2 A \times \Delta V \times S}
$$

where $C_{A}$ is the areal capacitance, $\int i d V$ is the integrated area of the $\mathrm{CV}$ curve, $A$ is the area of the electrode, $\Delta V$ is the potential range, and $S$ is the scan rate.

The maximum areal capacitance of $1964 \mathrm{mFcm}^{-2}$ is obtained for $\mathrm{ZnCo}-\mathrm{LDH}$ at a scan rate of $5 \mathrm{mVs}^{-1}$. The variation of areal capacitance with respect to scan rate is as shown in Fig. 4(b). It is well known that the capacitance decreases with an increasing scan rate. At low scan rates, there is sufficient time for ions to penetrate into pores to produce high charges and higher capacitance whereas at high scan rates, the electrolyte can hardly enter the pores, which reduces the capacitance. The areal capacitance of $\mathrm{ZnCo-LDH}$ decreased from 1964 to $527 \mathrm{mFcm}^{-2}$, with an increasing scan rate from 5 to $100 \mathrm{mVs}^{-1}$.

Figure 4(c) shows the galvanostatic charge discharge curves of ZnCo-LDH recorded over -0.1 to $0.5 \mathrm{~V}$ vs. SCE at different applied currents. The small deviation of the triangular shape of the charge discharge curves from the straight line further confirms that the capacitance of $\mathrm{ZnCo}-\mathrm{LDH}$ originated from the faradaic redox reversible reactions. With increasing applied currents, the voltage rapidly decreases; as a result, the discharge time reduces. The areal energy density (ED) and power density (PD) of the electrode were calculated from the Galvanostatic Charge-discharge (GCD) curves using the following equations:

$$
\begin{gathered}
E D=\frac{I t \triangle V}{A} \\
P D=\frac{I \triangle V}{A}
\end{gathered}
$$

where $I$ is the applied current, $t$ is the discharge time, $\Delta V$ is the potential range, and $A$ is the area of the electrode.

The maximum energy density of $0.22 \mathrm{mWcm}^{-2}$ and power density of $9 \mathrm{mWcm}^{-2}$ are achieved for $\mathrm{ZnCo}-\mathrm{LDH}$, at an applied current of $15 \mathrm{~mA}$.

The Electrochemical Impedance Spectroscopy measurement was performed to analyze the charge transfer and ion transport performance of the $\mathrm{ZnCo}-\mathrm{LDH}$ electrode. Figure 4(d) displays the Nyquist plot of the $\mathrm{ZnCo-LDH}$ electrode measured in the frequency range from 0.1 to $1 \times 10^{5} \mathrm{~Hz}$. The Nyquist plot is composed of two regions: a high frequency region (semi-circle) and a low frequency region (a straight line); this confirms the faradaic charge storage mechanism of the $\mathrm{ZnCo-LDH}$ electrode [14]. The intercept with the real axis at very high frequencies indicates the equivalent series resistance and the diameter of the semicircle is equal to the charge transfer resistance of the electrode [15]. From Fig. 4(d), an equivalent series resistance of $0.4 \Omega$ was obtained for $\mathrm{ZnCo}-\mathrm{LDH}$. The very small arc diameter was attributed to the fast ion diffusion and low charge transfer resistance of the $\mathrm{ZnCo}-\mathrm{LDH}$ [13]. The impedance phase angle vs. the frequency plot for the $\mathrm{ZnCo-} \mathrm{LDH}$ electrode is shown in the

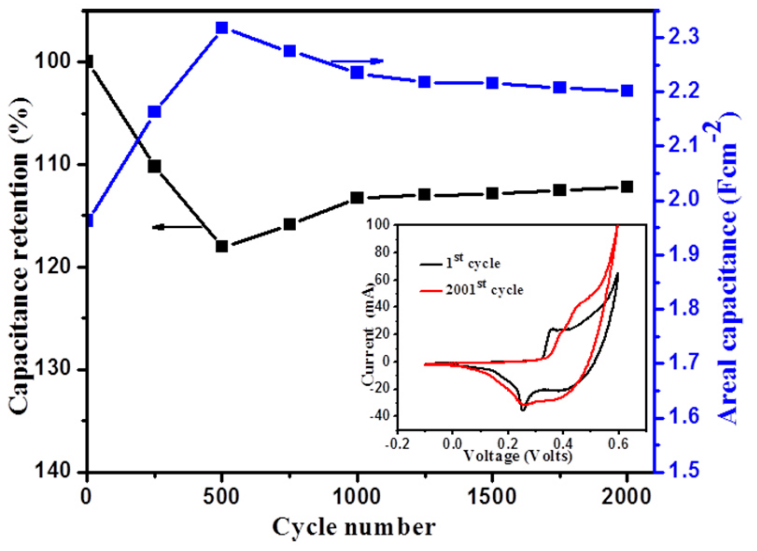

Figure 5. (Color online) Capacitance retention and areal capacitance versus cycle number. Inset: CV curves obtained at $1^{\text {st }}$ and $2001^{\text {st }}$ cycles at $5 \mathrm{mVs}^{-1}$.

inset of Fig. 4(d). The impedance phase angle for $\mathrm{ZnCo-LDH}$ was found to be $\sim 66.5^{\circ}$ at low frequency.

To evaluate the cycling performance of the $\mathrm{ZnCo-LDH}$ electrode, the electrode was subjected to 2000 cycles at a scan rate of $5 \mathrm{mVs}^{-1}$. Figure 5 represents the change in the capacitance retention and areal capacitance with the number of cycles. The inset in Fig. 5 denotes the CVs of the $1^{\text {st }}$ and $2001^{\text {st }}$ cycles recorded at $5 \mathrm{mVs}^{-1}$ for $\mathrm{ZnCo-LDH}$. An increase in the current was observed in the $\mathrm{CV}$ after performing 2000 cycles, which indicated the rise in electrochemical performance upon cycling. With an increase in the number of cycles, the areal capacitance reached a maximum of $2318 \mathrm{mFcm}^{-2}$, with $118 \%$ capacitance retention, after 500 electrochemical cycles. Furthermore, a $4.7 \%$ loss was observed in the capacitance for the next 500 cycles. Next, only a $1.1 \%$ loss was found for the next 1000 cycles, which indicates an almost stable electrochemical behavior of the electrode. That is, a total of $112.2 \%$ capacitance retention with $2202 \mathrm{mFcm}^{-2}$ areal capacitance was achieved after 2000 cycles, suggesting the stable and excellent performance of the $\mathrm{ZnCo}-\mathrm{LDH}$ electrode. This is attributed to the improved surface wetting and electrochemical activation of the $\mathrm{ZnCo}-$ $\mathrm{LDH}$ during the insertion and extraction of ions.

\section{Conclusions}

We fabricated $\mathrm{ZnCo}$ LDH nanostructures using a facile one pot hydrothermal synthesis. The interconnected hexagonal sheets having ultrathin thicknesses of $\mathrm{ZnCo} \mathrm{LDH} \mathrm{with} \mathrm{voids} \mathrm{are} \mathrm{highly} \mathrm{beneficial} \mathrm{for}$ the effective penetration of electrolytes leading to superior electrochemical properties. The as-synthesized $\mathrm{ZnCo} \mathrm{LDH}$ nanostructure produced an areal capacitance of $1964 \mathrm{mFcm}^{-2}$ at $5 \mathrm{mVs}^{-1}$ scan rate, energy density of $0.22 \mathrm{mWhcm}^{-2}$, and excellent cycling stability (112 $\%$ ) after 2000 charge discharge cycles. This study provides LDH ZnCo bimetallic hydroxide as a promising electrode for efficient pseudocapacitors, and it opens an avenue for the use of different bimetallic LDHs as an electrode material for electrochemical supercapacitors.

\section{Acknowledgements}

This study was supported by the National Research Foundation of Korea (NRF-2017R1C1B2010906 and NRF-2017M1A2A2048904). 


\section{References}

[1] P. Simon and Y. Gogotsi, Nat. Mater. 7, 845 (2008).

[2] R. Kötz and M. Carlen, Electrochim. Acta 45, 2483 (2000).

[3] C. Liu, Z. Yu, D. Neff, A. Zhamu, and B. Z. Jang, Nano Lett. 10, 4863 (2010).

[4] C. Q. Yi, J. P. Zou, H. Z. Yang, and X. Leng, Trans. Nonferrous Met. Soc. China 28, 1980 (2018).

[5] H. Zhao, M. Zhou, L. Wen, and Y. Lei, Nano Energy 13, 790 (2015).

[6] M. Shao, F. Ning, Y. Zhao, J. Zhao, M. Wei, D. G. Evans, and X. Duan, Chem. Mater. 24, 1192 (2012).

[7] Q. Zhang and F. Wei, Eds., Advanced Hierarchical Nanostructured Materials (Wiley-VCH Verlag GmbH \& Co. KGaA, Weinheim, Germany, 2014).

[8] X. Bai, J. Liu, Q. Liu, R. Chen, X. Jing, B. Li, and J. Wang, Chem. A
Eur. J. 23, 14839 (2017).

[9] J. Chen, X. Wang, J. Wang, and P. S. Lee, Adv. Energy Mater. 6, 1501745 (2016).

[10] T. Liang, H. Xuan, Y. Xu, J. Gao, X. Han, J. Yang, P Han, D. Wang, and Y. Du, ChemElectroChem 5, 2424 (2018).

[11] D. Xu, Y. Rui, Y. Li, Q. Zhang, and H. Wang, Appl. Surf. Sci. 358, 436 (2015).

[12] M. Wang, L. Jiang, E. J. Kim, and S. H. Hahn, RSC Adv. 5, 87496 (2015).

[13] D. S. Patil, S. A. Pawar, and J. C. Shin, Chem. Eng. J. 335, 693 (2018).

[14] V. Venkatachalam, A. Alsalme, A. Alswieleh, and R. Jayavel, Chem. Eng. J. 321, 474 (2017).

[15] D. S. Patil, S. A. Pawar, J. C. Shin, and H. J. Kim, J. Power Sources 435, 226812 (2019). 